COMMUNICATIONS IN

ANALYSIS AND GEOMETRY

Volume 12, Number 1, 281-303, 2004

\title{
Potential Functions and Actions of Tori on Kähler Manifolds
}

\author{
D. Burns And V. Guillemin
}

\section{Introduction.}

Let $M$ be a Kähler manifold equipped with a free Hamiltonian action of the standard $n$-torus, $T$ with moment map, $\Phi: M \rightarrow \mathbb{R}^{n}$. For $\lambda \in \mathbb{R}^{n}$ the symplectic quotient

$$
M_{\lambda}=\Phi^{-1}(\lambda) / T
$$

inherits from $M$ a Kähler structure, and in the first part of this paper ${ }^{2}$ we will describe what the Kähler form and Ricci form look like locally on coordinate patches in $M_{\lambda}$. Then in the second part of this paper we will discuss some global implications of these results. This will include

1. A Kählerian proof of the Duistermaat-Heckman theorem.

2. A formula, due to Biquard and Gauduchon, for the Kähler potential on a symplectic quotient.

3. A convexity theorem of Atiyah for moment images of $T_{\mathbb{C}^{-} \text {-orbits. }}$

4. A formula in terms of moment data for the Kähler metric on a toric variety.

5. A formula for the Kähler form on the symplectic quotient of a KählerEinstein manifold.

A few comments about each of these items:

\footnotetext{
${ }^{1}$ Supported in part by NSF grants DMS-0104047 (DB) and DMS-0104116 (VG).

${ }^{2}$ Note added in proof. Most of the results of the first part are already known: see the detailed remark at the end of section 1 below.
} 
1. The usual proof of the Duistermaat-Heckman theorem is global and topological in nature. Our proof in the Kähler case consists of showing that locally on a coordinate patch in $M_{\lambda}$ two canonically defined Kähler forms are identical.

2. The formula of Biquard-Gauduchon was used by Calderbank, David and Gauduchon to give an elegant economical proof of the theorem alluded to in item 4 . (This theorem is due to one of us, and a much less elegant proof of it was presented in $[\mathrm{Gu}]$.) In this paper we use a primitive local form of the Biquard-Gauduchon theorem (see $\S 4$, formula (4.5)) to give an even simpler derivation of this formula.

3. Atiyah's proof of this convexity theorem for $T_{\mathbb{C} \text {-orbits involves a care- }}$ ful analysis of the global geometry of the closures of these orbits. The proof we present here is quite different in spirit. In the spirit of this article it is based on convexity properties of strictly pluri-subharmonic potentials. Unfortunately, we must assume that the Kähler form defines an integral cohomology class, an hypothesis Atiyah avoids. Although standard approximation techniques will eliminate this hypothesis in some cases, we are unable to derive Atiyah's theorem in full generality.

4. It is well-known that the symplectic quotient by a circle action of a Kähler-Einstein manifold is not in general Kähler-Einstein. (See, for instance, $[\mathrm{Be}]$ and $[\mathrm{Fu}] \S 7.3^{1}$.) As was pointed out to us by Michael Duff, this is analogous to what happens, vis a vis dimensional reduction by circle actions, in general relativity. By Kaluza-Klein, the quotient by a circle action of a $4+1$-dimensional Einstein manifold has, in addition to its downstairs metric, a two-form (magnetic field) and an electrostatic potential; and the field equations satisfied by these data are not just the field equations for gravity alone, but for gravity coupled with electromagnetism. In other words the field equations on the quotient space reflect the fact that the geometry of the quotient space is more complicated than the geometry of the original space.

We will show that this is true for symplectic quotients as well. For $\lambda \in \mathbb{R}^{n}$, the level set, $\Phi^{-1}(\lambda)$, is a principal $T$-bundle over $M_{\lambda}$, and the metric on $M$ gives one a connection on this bundle and a set of curvature forms, $\mu_{i}$. In addition one gets from the metric a potential

\footnotetext{
${ }^{1}$ We take this opportunity to remark that the examples of non-existence of extremal metrics on ruled surfaces cited in [Fu] as "Burns's examples" are due to P. DeBartolomeis and one of us [BD]
} 
function on $M_{\lambda}$, the effective potential: $V_{\text {eff }}$, whose value at $p \in M_{\lambda}$ is the volume of the fiber above $p$ in $\Phi^{-1}(\lambda) .{ }^{2}$ We will show that if the Kähler form, $\omega$, and the Ricci form, $\mu$, satisfy on $M$ the KählerEinstein equation, $\mu=\kappa \omega$, then the reduced Kähler form, $\omega_{\lambda}$, and reduced Ricci form, $\mu_{\lambda}$, satisfy

$$
\mu_{\lambda}-2 \sqrt{-1} \partial \bar{\partial} \log V_{\text {eff }}+\sum c_{i} \mu_{i}=\kappa\left(\omega_{\lambda}+\sum \lambda_{i} \mu_{i}\right)
$$

the $c_{i}$ 's being constants which don't depend on $\lambda$. This identity can be thought of as a Kähler version of the Einstein equation for a metric coupled with an electromagnetic field.

The presence of the $c_{i}$ 's in (1.1) is due, incidentally, to the fact that the moment map is only well-defined up to an additive constant. We will show in $\S 11$ that, for one very natural normalization of this constant, the $c_{i}$ 's are equal to zero. The proof of this will depend on a fact about Kähler-Einstein manifolds which is of independent interest. Let $Z_{i}, i=1, \ldots, n$, be the complex vector fields generating the action of $T_{\mathbb{C}}$ on $M$. Then the components, $\phi_{i}$, of the moment map satisfy the divergence identites

$$
\kappa \phi_{i}=\operatorname{div} Z_{i}+c_{i} .
$$

We remark that equations like 1.1 have been exploited earlier by $\mathrm{C}$. LeBrun and coworkers for the construction of Kähler-Einstein and constant scalar curvature surfaces: see especially $[\mathrm{L}]$ and $[\mathrm{PP}]$.

Finally, we thank Peter Heinzner and Alan Huckleberry for pointing out to us that the basics of reduction in terms of potentials in the first part of the paper have been carried out already in [HHL], [HL] and [S], (which are conveniently reviewed in [HH2] and [HH3]). We treat here only the case of a free torus action. In particular, we also note that Kähler potentials have already been used by them to prove convexity theorems for more general moment maps in [HH1].

\section{The Darboux theorem for $T$-invariant Kähler forms.}

Let $\mathbb{C}^{*}=\mathbb{C}-\{0\}$ and let $T_{\mathbb{C}}=\left(\mathbb{C}^{*}\right)^{n}$ be the complexification of the group, $T=\left(S^{1}\right)^{n}$. Let $U$ be an open convex subset of $\mathbb{C}^{m}$ and let

$$
M=T_{\mathbb{C}} \times U=\left(\mathbb{C}^{*}\right)^{n} \times U
$$

\footnotetext{
${ }^{2}$ For circle actions, this coincides with the usual "effective potential" for reduced systems in classical mechanics. (See $[\mathrm{AM}], \S 4.5$.)
} 


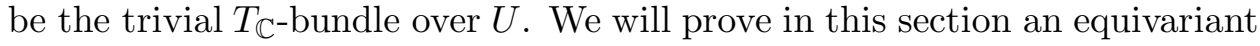
Darboux theorem for Kähler forms on $M$. Let $\omega$ be a $T$-invariant Kähler form which is Hamiltonian with respect to the action of $T$; and let $z_{1}, \ldots, z_{n}$ and $w_{1}, \ldots, w_{m}$ be the coordinate functions on $\left(\mathbb{C}^{*}\right)^{n}$ and $U$. We will prove that

$$
\omega=\sqrt{-1} \partial \bar{\partial} \rho
$$

where

$$
\rho=\rho\left(t_{1}, \ldots, t_{n}, w_{1}, \ldots, w_{m}\right), \quad t_{i}=\left|z_{i}\right|^{2} .
$$

Moreover, we will show that if $\rho_{1}$ and $\rho_{2}$ are two such functions

$$
\rho_{2}-\rho_{1}=\sum \lambda_{i} \log t_{i}+\operatorname{Re} f
$$

with $\lambda_{i} \in \mathbb{R}$ and $f \in \mathcal{O}(U)$.

Proof. Since the action of $T$ is Hamiltonian the orbits of $T$ are isotropic. However, for $p_{0} \in M$, the orbit, $T \cdot p_{0}$ is a deformation retract of $M$. Therefore, since the restriction of $\omega$ to this orbit is zero, $\omega$ is exact; i.e., $\omega=d \alpha$. Let $\alpha=\alpha_{0,1}+\alpha_{1,0}$. Then, $\omega=\partial \alpha_{0,1}+\bar{\partial} \alpha_{1,0}$; and $\bar{\partial} \alpha_{0,1}=\partial \alpha_{1,0}=0$. Since $M$ is a domain of holomorphy, $\alpha_{0,1}=\bar{\partial} h$ and hence

$$
\omega=\partial \bar{\partial}(h-\bar{h})=\sqrt{-1} \partial \bar{\partial} \rho
$$

where $\sqrt{-1} \rho=h-\bar{h}$. By averaging with respect to $T$ we can assume that $\rho$ is $T$-invariant and hence of the form (2.3). To prove the assertion (2.4) we make use of the following elementary fact.

Lemma 2.1. If $u \in C^{\infty}\left(\mathbb{C}^{*}\right)$ is a radially symmetric harmonic function it is of the form $c_{1} \log r+c_{2}, c_{i} \in \mathbb{C}$.

Proof. By hypothesis

$$
\Delta u=\frac{d^{2}}{d r^{2}} u+\frac{1}{r} \frac{d u}{d r}=0,
$$

and all solutions of this ODE are of the form above.

We will now prove (2.4) by induction on $n$. The difference, $g=\rho_{2}-\rho_{1}$, is harmonic in $z_{1}$, so it has to be of the form

$$
g_{1}\left(z^{\prime}, w\right) \log \left|z_{1}\right|^{2}+g_{2}\left(z^{\prime}, w\right), \quad z^{\prime}=\left(z_{2}, \ldots, z_{n}\right) .
$$


However,

$$
0=\frac{\partial^{2}}{\partial z_{1} \partial \bar{z}_{i}} g=\frac{1}{z_{1}} \frac{\partial}{\partial \bar{z}_{i}} g_{1}\left(z^{\prime}, w\right),
$$

so the derivative of $g_{1}$ with respect to $\bar{z}_{i}$ is zero and, since $g_{1}$ is real-valued, so is the derivative with respect to $z_{i}$. Similarly the derivatives of $g_{1}$ with respect to $w_{i}$ and $\bar{w}_{i}$ are zero; so $g_{1}$ is constant. Thus

$$
g=\lambda_{1} \log \left|z_{1}\right|^{2}+g_{2}\left(z^{\prime}, w\right) .
$$

Now apply induction.

The result we have just proved can be restated:

Theorem 2.2. If $g$ is a T-invariant real-valued pluri-harmonic function on $M$ then $g$ is of the form

$$
\sum \lambda_{i} \log t_{i}+\operatorname{Re} f
$$

with $\lambda_{i} \in \mathbb{R}$ and $f \in \mathcal{O}(U)$.

\section{The moment map.}

We will compute below the moment map associated with the action of $T$ on $M$. Let $\rho$ be the potential function (2.3). Letting $\alpha=-i \partial \rho$, one gets from (2.1), where $M$ is as in $\S 2$.

$$
\omega=d \alpha
$$

and hence with

$$
\frac{\partial}{\partial \theta_{j}}=i\left(z_{j} \frac{\partial}{\partial z_{j}}-\bar{z}_{j} \frac{\partial}{\partial \bar{z}_{j}}\right)
$$

one has

$$
\iota\left(\frac{\partial}{\partial \theta_{j}}\right) \omega=-d\left(\iota\left(\frac{\partial}{\partial \theta_{j}}\right) \alpha\right)
$$

so we get for the $j^{\text {th }}$ component of the moment map

$$
\phi_{j}=\iota\left(\frac{\partial}{\partial \theta_{i}}\right) \alpha=z_{j} \frac{\partial \rho}{\partial z_{j}}=t_{j} \frac{\partial \rho}{\partial t_{j}} .
$$

Unlike the symplectic form, $\omega,(3.1)$ depends upon the choice of $\rho$. If we replace $\rho$ by the potential function

$$
\rho_{1}=\rho+\sum \lambda_{j} \log t_{i}+\operatorname{Re} f \quad f \in \mathcal{O}(U)
$$


and set

$$
\alpha_{1}=-i \partial \rho_{1}=-i \partial \rho-i \sum \lambda_{j} \frac{d z_{j}}{z_{j}}-\frac{i}{2} \partial f
$$

we get for the $j^{\text {th }}$ component of the moment map

$$
\iota\left(\frac{\partial}{\partial \theta_{j}}\right) \alpha_{1}=\iota\left(\frac{\partial}{\partial \theta_{j}}\right) \alpha+\lambda_{j}
$$

i.e., the moment map associated with $\rho_{1}$ is

$$
\Phi_{1}=\Phi+\lambda
$$

Thus if $\Phi$ and $\Phi_{1}$ are the same, $\lambda=0$ and hence by (3.2) $\rho_{1}=\rho+\operatorname{Re} f$, $f \in \mathcal{O}(U)$. Thus to summarize, we proved

Theorem 3.1. Let $\rho_{1}$ and $\rho$ be strictly pluri-subharmonic functions of the form (2.3). If the symplectic forms and moment maps associated with $\rho$ and $\rho_{1}$ are the same then

$$
\rho_{1}=\rho+\operatorname{Re} f, \quad f \in \mathcal{O}(U) .
$$

Coming back to the formula (3.1) note that if we make the change of variables $t_{i}=e^{s_{i}}$ and set

$$
\rho\left(t_{1}, \ldots, t_{n}, w\right)=\rho\left(e^{s_{1}}, \ldots, e^{s_{n}}, w\right)=: f\left(s_{1}, \ldots s_{n}, w\right)
$$

the moment map can be written in the simpler form

$$
\phi_{j}(s, w)=\frac{\partial}{\partial s_{j}} f(s, w) .
$$

\section{The reduced potential function.}

As above let $\rho$ be the potential function (2.3) and let

$$
\alpha=-i \partial \rho=-i \sum \frac{\partial \rho}{\partial z_{j}} d z_{j}+\partial_{w} \rho .
$$

By $(3.1), z_{j} \partial \rho / \partial z_{j}$ is equal to $\phi_{j}$; hence

$$
\alpha=-i\left(\sum \phi_{j} d \log z_{j}+\partial_{w} \rho\right) .
$$


Let us denote by $\iota_{\lambda}$ the inclusion of the level set $\phi^{-1}(\lambda)$ and by $\pi$ the projection of $T_{\mathbb{C}} \times U$ onto $U$. The composite map $\pi \circ \iota_{\lambda}$ is $T$-invariant and gives us an identification locally of $U$ with the reduced space

$$
M_{\lambda}=\phi^{-1}(\lambda) / T
$$

and via this identification, $w_{1}, \ldots, w_{n}$ become local holomorphic coordinates on $M_{\lambda}$.

From the identity 4.1, we get

$$
\iota_{\lambda}^{*} \alpha=-i \iota_{\lambda}^{*} \partial_{w} \rho+d h_{\lambda}
$$

where $h_{\lambda}=-i \sum \lambda_{j} \log \iota_{\lambda}^{*} z_{j}$. Setting $\iota_{\lambda}^{*} t_{j}=t_{j}(w)$, one can interchange $\iota_{\lambda}^{*}$ and $\partial_{w}$ :

$$
\iota_{\lambda}^{*} \frac{\partial}{\partial w} \rho(t, w)=\frac{\partial}{\partial w} \rho(t(w), w)-\sum \frac{\partial \rho}{\partial t_{j}} \frac{\partial t_{j}}{\partial w},
$$

and, by (3.1), rewrite this in the form

$$
\iota_{\lambda}^{*} \frac{\partial}{\partial w} \rho(t, w)=\frac{\partial}{\partial w}\left(\iota_{\lambda}^{*} \rho-\sum \lambda_{j} \log \iota_{\lambda}^{*} t_{j}\right) .
$$

Hence (4.2) becomes

$$
\iota_{\lambda}^{*} \alpha=-i \partial_{w} \iota_{\lambda}^{*}\left(\rho-\sum \lambda_{j} \log t_{j}\right)+d h_{\lambda},
$$

so the reduced symplectic form $d \iota_{\lambda}^{*} \alpha$ on $M_{\lambda}$ is equal to

$$
\omega_{\lambda}=i \partial_{w} \bar{\partial}_{w} \rho_{\lambda}
$$

where

$$
\rho_{\lambda}=\iota_{\lambda}^{*}\left(\rho-\sum \lambda_{j} \log t_{i}\right)
$$

\section{The reduced Kähler metric on $M_{\lambda}$.}

By equation (4.5)

$$
\omega_{\lambda}=i \sum \psi_{\alpha, \bar{\beta}} d w_{\alpha} \wedge d \bar{w}_{\beta}
$$

where

$$
\psi_{\alpha, \bar{\beta}}=\frac{\partial^{2} \rho_{\lambda}}{\partial w_{\alpha} \partial \bar{w}_{\beta}}
$$


To compute these derivatives we will, as in (3.5), set $t_{i}=e^{s_{i}}$ and let

$$
f\left(s_{1}, \ldots, s_{n}, w\right)=\rho\left(t_{1}, \ldots, t_{n}, w\right) .
$$

In these coordinates the $\lambda$-level set of the moment map is defined by the equations

$$
\frac{\partial f}{\partial s_{i}}=\lambda_{i}
$$

so if we let

$$
g(s, w)=f(s, w)-\sum \lambda_{i} s_{i}
$$

then, by (4.5), $\rho_{\lambda}$ is the restriction of $g$ to the set

$$
\frac{\partial g}{\partial s_{i}}=0
$$

Letting $s=s(w)$ on this set, we obtain from (5.2) and (4.5):

$$
\psi_{\alpha, \bar{\beta}}=\frac{\partial}{\partial w_{\alpha}} \frac{\partial}{\partial \bar{w}_{\beta}}(g(s(w), w)) .
$$

However, by (5.4)

$$
\frac{\partial}{\partial w_{\alpha}}(g(s(w), w))=\frac{\partial g}{\partial w_{\alpha}}(s(w), w)
$$

and hence

$$
\psi_{\alpha, \bar{\beta}}=\sum\left(\iota_{\lambda}^{*} \frac{\partial^{2} g}{\partial s_{i} \partial w_{\alpha}}\right) \frac{\partial s_{i}}{\partial \bar{w}_{\beta}}+\iota_{\lambda}^{*} \frac{\partial^{2} g}{\partial w_{\alpha} \partial \bar{w}_{\beta}} .
$$

Moreover, by (5.4)

$$
\frac{\partial}{\partial w_{\alpha}}\left(\frac{\partial g}{\partial s_{i}}(s(w), w)\right)=0=\sum_{j} \frac{\partial^{2} g}{\partial s_{i} \partial s_{j}}(s(w), w)+\frac{\partial^{2} g}{\partial s_{i} \partial w_{\alpha}}(s(w), w) .
$$

Hence

$$
\iota_{\lambda}^{*} \frac{\partial^{2} g}{\partial s_{i} \partial w_{\alpha}}=-\sum\left(\iota_{\lambda}^{*} \frac{\partial^{2} g}{\partial s_{i} \partial s_{j}}\right) \frac{\partial s_{j}}{\partial w_{\alpha}}
$$

and if we substitute this into (5.5) we get the following slightly more symmetric formula for $\psi_{\alpha, \bar{\beta}}$ :

$$
\psi_{\alpha, \bar{\beta}}=\iota_{\lambda}^{*} \frac{\partial^{2} g}{\partial w_{\alpha} \partial \bar{w}_{\beta}}-\sum\left(\iota_{\lambda}^{*} \frac{\partial^{2} g}{\partial s_{i} \partial s_{j}}\right) \frac{\partial s_{i}}{\partial w_{\alpha}} \frac{\partial s_{j}}{\partial \bar{w}_{\beta}} .
$$




\section{The Ricci potential.}

By definition the Ricci potential of the Kähler form, $i \partial \bar{\partial} \rho$, is minus the log determinant of the matrix

$$
\left[\begin{array}{cc}
\frac{\partial^{2} \rho}{\partial z_{i} \partial \bar{z}_{j}} & \frac{\partial^{2} \rho}{\partial z_{i} \partial \bar{w}_{\beta}} \\
\frac{\partial^{2} \rho}{\partial w_{\alpha} \partial \bar{z}_{j}} & \frac{\partial^{2} \rho}{\partial w_{\alpha} \partial \bar{w}_{\beta}}
\end{array}\right] .
$$

But with $s_{i}=\log \left|z_{i}\right|^{2}$

$$
\begin{aligned}
\frac{\partial^{2} \rho}{\partial z_{i} \partial \bar{z}_{j}} & =\frac{\partial^{2}}{\partial z_{i} \partial \bar{z}_{j}}\left(\rho-\sum \lambda_{\kappa} \log \left|z_{\kappa}\right|^{2}\right) \\
& =\frac{\partial^{2}}{\partial z_{i} \partial \bar{z}_{j}} g(s, w)=\frac{1}{z_{i} \bar{z}_{j}} \frac{\partial^{2} g}{\partial s_{i} \partial s_{j}} .
\end{aligned}
$$

Similarly

$$
\begin{aligned}
\frac{\partial^{2} \rho}{\partial z_{i} \partial \bar{w}_{\beta}} & =\frac{1}{z_{i}} \frac{\partial^{2} g}{\partial s_{i} \partial \bar{w}_{\beta}} \\
\frac{\partial^{2} \rho}{\partial w_{\alpha} \partial \bar{z}_{j}} & =\frac{1}{\bar{z}_{j}} \frac{\partial^{2} g}{\partial w_{\alpha} \partial s_{j}}
\end{aligned}
$$

and

$$
\frac{\partial^{2} \rho}{\partial w_{\alpha} \partial \bar{w}_{\beta}}=\frac{\partial^{2} g}{\partial w_{\alpha} \partial \bar{w}_{\beta}}
$$

so the determinant of the matrix above is equal to the determinant of the matrix

$$
\left[\begin{array}{cc}
\frac{\partial^{2} g}{\partial s_{i} \partial s_{j}} & \frac{\partial^{2} g}{\partial s_{i} \partial \bar{w}_{\beta}} \\
\frac{\partial^{2} g}{\partial w_{\alpha} \partial s_{j}} & \frac{\partial^{2} g}{\partial w_{\alpha} \partial \bar{w}_{\beta}}
\end{array}\right]
$$

times a factor of

$$
\frac{1}{\left|z_{1}\right|^{2} \cdots\left|z_{n}\right|^{2}}=e^{-\left(s_{1}+\cdots+s_{n}\right)} .
$$

Now consider the restriction of this matrix to the set, $\Phi^{-1}(\lambda)$. If we multiply the $j^{\text {th }}$ column of this matrix by $\partial s_{j} / \partial \bar{w}_{\beta}$ and subtract it from the $\beta^{\text {th }}$ 
column we get by (5.5) and (5.6) the matrix

$$
\left[\begin{array}{cc}
\frac{\partial^{2} g}{\partial s_{i} \partial s_{j}} & 0 \\
\cdots & \psi_{\alpha, \bar{\beta}}
\end{array}\right]
$$

Hence the determinant of the matrix $(*)$ is

$$
e^{-\left(s_{1}+\cdots+s_{n}\right)} \operatorname{det}\left(\frac{\partial^{2} g}{\partial s_{i} \partial s_{j}}\right) \operatorname{det}\left(\psi_{\alpha, \bar{\beta}}\right)
$$

or

$$
\operatorname{det}\left(\frac{\partial^{2} \rho}{\partial z_{i} \partial \bar{z}_{j}}\right) \operatorname{det}\left(\psi_{\alpha, \bar{\beta}}\right) \text {. }
$$

Thus we've shown:

Theorem 6.1. Let h be the Ricci potential of the Kähler form, $\omega$. Then the Ricci potential, $h_{\lambda}$, of the Kähler form $\omega_{\lambda}$ is

$$
h_{\lambda}=\iota_{\lambda}^{*}\left(h+\log \operatorname{det}\left(\frac{\partial^{2} \rho}{\partial z_{i} \partial \bar{z}_{j}}\right)\right) .
$$

The second summand on the right has a simple geometric interpretation. Let $\nu_{i}=d \log z_{i}$. At a point $p \in M$ the restriction of $\omega$ to the orbit, $T_{\mathbb{C}} \cdot p$, is equal to

$$
\sqrt{-1} \sum z_{i} \bar{z}_{j} \frac{\partial^{2} \rho}{\partial z_{i} \partial \bar{z}_{j}} \nu_{i} \wedge \bar{\nu}_{j}
$$

and the Kählerian volume form on this orbit is

$$
\sqrt{-1} f \nu_{1} \wedge \bar{\nu}_{1} \wedge \cdots \wedge \nu_{n} \wedge \bar{\nu}_{n}
$$

where

$$
f=2^{n}\left|z_{1}\right|^{2} \ldots\left|z_{n}\right|^{2} \operatorname{det}\left(\frac{\partial^{2} \rho}{\partial z_{i} \partial \bar{z}_{j}}\right) .
$$

But by (6.7) the map

$$
J_{p}: \operatorname{Re} \nu_{i} \rightarrow \operatorname{Im} \nu_{i}, i=1, \ldots, n
$$

maps the space

$$
\operatorname{span}\left\{\operatorname{Re} \nu_{i}, i=1, \ldots, n\right\}
$$

at $p$ isometrically onto the space

$$
\operatorname{span}\left\{\operatorname{Im} \nu_{i}, i=1, \ldots, n\right\} .
$$


Moreover, (6.7) also implies that these spaces are Riemannian orthocomplements of each other in the cotangent space at $p$ to $T_{\mathbb{C}} \cdot p$. Hence if $g \operatorname{Re} \nu_{1} \wedge \cdots \wedge \operatorname{Re} \nu_{n}$ is the Riemannian volume form on the space (6.10), $g \operatorname{Im} \nu_{1} \wedge \cdots \wedge \operatorname{Im} \nu_{n}$ is the Riemannian volume form on the space (6.11); and the wedge product of these two forms is the Riemannian volume form on the cotangent space at $p$ to $T_{\mathbb{C}} \cdot p$, which is just the volume form (6.8). Thus $g^{2}=f$. Moreover, the space (6.11) is just the cotangent space to $T \cdot p$ at $p$ and $\operatorname{Im} \nu_{i}=d \theta_{i}$. Hence the volume form on the $T$ orbit through $p$ is $\sqrt{f} d \theta_{1} \wedge \cdots \wedge d \theta_{n}$ and the volume of this orbit is $(2 \pi)^{n} \sqrt{f(p)}$. Thus $\iota_{\lambda}^{*} \sqrt{f}$ is the effective potential, $V_{\text {eff }}$, that we defined in $\S 1$; and, up to an additive constant,

$$
h_{\lambda}=\iota_{\lambda}^{*}\left(h+\sum \log \left|z_{i}\right|^{2}\right)+2 \log V_{\text {eff }}
$$

\section{GIT quotients.}

In the second half of this paper we will describe some global applications of the results of $\S \S 1-6$. Let $M$ now be a $d$-dimensional compact complex manifold, and $\tau: T_{\mathbb{C}} \times M \rightarrow M$ a holomorphic action of $T_{\mathbb{C}}$ on $M$. Let $\omega \in \Omega^{1,1}(M)$ be a Kähler form with respect to which the restriction of $\tau$ to $T$ is Hamiltonian and let $\Phi: M \rightarrow \mathbb{R}$ be the moment map, normalized so that

$$
\int \phi^{i} \omega^{d}=0
$$

We will assume that 0 is a regular value of $\Phi$ and that $T$ acts freely on the level set $\Phi^{-1}(0)$. Then, for $\lambda$ close to $0, T$ acts freely on $\Phi^{-1}(\lambda)$. Hence the reduced space

$$
M_{\lambda}=\Phi^{-1}(\lambda) / T
$$

is well-defined and is a compact Kähler manifold. We will denote by $\omega_{\lambda}$ its Kähler form.

We recall (see $[\mathrm{MFK}]$ ) that the set of stable points in $M$ is the set

$$
M_{\text {stable }}=T_{\mathbb{C}} \cdot \Phi^{-1}(0) .
$$

This set is an open dense subset of $M$ whose complement is a subvariety of dimension $\leq d-1$. Moreover, $T_{\mathbb{C}}$ acts freely and properly on $M_{\text {stable }}$, so the quotient space

$$
M_{\text {red }}=M_{\text {stable }} / T_{\mathbb{C}}
$$

is a compact complex manifold of dimension $\ell=d-n$, and, by definition, $M_{\text {red }}$ is the geometric invariant theory (GIT) quotient of $M$ by $T_{\mathbb{C}}$. The 
projection

$$
\pi: M_{\text {stable }} \rightarrow M_{\text {red }}
$$

makes $M_{\text {stable }}$ into a principal $T_{\mathbb{C}}$ bundle over $M_{\text {red }}$. Moreover, since

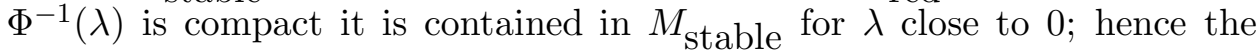
map

$$
\Phi^{-1}(\lambda) \hookrightarrow M_{\text {stable }} \stackrel{\pi}{\longrightarrow} M_{\text {red }}
$$

induces a biholomorphic map, $M_{\lambda} \rightarrow M_{\text {red }}$; so, as complex manifolds, the $M_{\lambda}$ 's are identical with $M_{\text {red }}$ and we can think of the $\omega_{\lambda}$ 's as a family of Kähler forms on $M_{\text {red }}$. In the first of our applications we will describe how the $\omega_{\lambda}$ 's vary as one varies the parameter, $\lambda$. In all the applications below, by the way, except for the application discussed in $\S 10$, we will only be concerned with the open subset, $M_{\text {stable, of }} M$ and the fibration (7.1). Moreover, for the most part, it won't be important that $M_{\text {red }}$ be compact; in other words, in most of the applications below, we can assume that $M=M_{\text {stable }}$ and allow $M$ to be a principal $T_{\mathbb{C}}$-bundle over a (not necessarily compact) base manifold, $M_{\text {red }}$.

\section{The Kähler version of Duistermaat-Heckman.}

Let $U$ be a convex coordinate patch in $M_{\text {red }}$ and let $M_{U}=\pi^{-1}(U)$. We will assume that the bundle (7.1) is trivial over $U$ and hence

$$
M_{U}=\left(\mathbb{C}^{*}\right)^{n} \times U .
$$

Let $\rho$ be a potential function on $M_{U}$ of the form (2.3) such that $\omega=\sqrt{-1} \partial \bar{\partial} \rho$ and such that the moment map (3.1) coincides with the moment map defined in the previous section. By (4.6) the reduced symplectic form on $U$ is equal to

$$
\sqrt{-1} \partial \bar{\partial} \iota_{\lambda}^{*} \rho-\sum \sqrt{-1} \lambda_{j} \partial \bar{\partial} \log \iota_{\lambda}^{*}\left|z_{j}\right|^{2} .
$$

(Here and throughout this section, we identify $T$-invariant functions on $\phi^{-1}(\lambda)$ with functions on $U$, i.e., as functions of the $w$ coordinates, where we can express, along $\phi^{-1}(\lambda),\left|z_{i}\right|^{2}=\left|z_{i}\right|^{2}(w)$.) We claim that the first term in (8.2) is an intrinsically defined 2-form on $U$ not depending on the trivialization (8.1). In fact if $\rho_{1}$ is another potential function with the same properties as $\rho$ then by Theorem 3.1, $\rho_{1}=\rho+\operatorname{Re} f$, for $f \in \mathcal{O}(U)$ and hence

$$
\sqrt{-1} \partial \bar{\partial} \iota_{\lambda}^{*} \rho_{1}=\sqrt{-1} \partial \bar{\partial} \iota_{\lambda}^{*} \rho .
$$

The same argument shows that for different values, $\lambda_{1}$ and $\lambda_{2}$, of $\lambda$

$$
\sqrt{-1} \bar{\partial} \iota_{\lambda_{1}}^{*} \rho-\sqrt{-1} \bar{\partial} \iota_{\lambda_{2}}^{*} \rho
$$


is an intrinsically defined one-form not depending on $\rho$; for if we replace $\rho$ by $\rho+\operatorname{Re} f$, this difference is unchanged. Thus there exists a globaly defined two-form, $\mu_{\lambda}$, whose restriction to $U$ satisfies

$$
\mu_{\lambda}=\sqrt{-1} \partial \bar{\partial} \iota_{\lambda}^{*} \rho
$$

and a globally defined one-form, $\nu_{1,2}$, whose restriction to $U$ satisfies

$$
\nu_{1,2}=\sqrt{-1} \bar{\partial} \iota_{\lambda_{1}}^{*} \rho-\sqrt{-1} \bar{\partial} \iota_{\lambda_{2}}^{*} \rho
$$

such that

$$
\mu_{\lambda_{1}}-\mu_{\lambda_{2}}=d \nu_{1,2} \text {. }
$$

Next consider the terms in the second summand of (8.2). The projection of the product, $T_{\mathbb{C}}=\left(\mathbb{C}^{*}\right)^{n}$ onto its $i^{\text {th }}$ factor defines a character

$$
\chi_{i}: T_{\mathbb{C}} \rightarrow \mathbb{C}^{*} .
$$

Let $\mathbb{L}_{i}$ be the line bundle over $M_{\text {red }}$ associated with this character: A section of this line bundle is by definition a map

$$
s: M_{\text {stable }} \rightarrow \mathbb{C}
$$

satisfying

$$
s(a p)=\chi_{i}(a)^{-1} s(p) ;
$$

so in particular the functions

$$
s_{i}: M_{U} \rightarrow \mathbb{C}^{*},(z, w) \rightarrow z_{i}^{-1}
$$

are non-vanishing holomorphic sections of $\mathbb{L}_{i}$ over $U$.

Given a map $s: M_{\text {stable }} \rightarrow \mathbb{C}$ satisfying (8.8) the restriction of $|s|^{2}$ to $\Phi^{-1}(\lambda)$ is, by (8.8) constant on the fibers of the $T$-bundle:

$$
\Phi^{-1}(\lambda) \rightarrow M_{\text {red }}
$$

so one can define a hermitian metric on $\mathbb{L}_{i}$ by setting

$$
\langle s, s\rangle_{\lambda}=\iota_{\lambda}^{*}|s|^{2},
$$

and in terms of this metric the $i^{\text {th }}$ summand of (8.2) is equal to

$$
-\sqrt{-1} \partial \bar{\partial} \log \left\langle s_{i}, s_{i}\right\rangle_{\lambda}=\mu_{i, \lambda}
$$

which is just the curvature form $\mu_{i, \lambda}$ of the bundle $\mathbb{L}_{i}$. This form depends on $\lambda$, however if $\lambda_{1}$ and $\lambda_{2}$ are distinct values of $\lambda$, the inner products, $\langle\cdot, \cdot\rangle_{\lambda_{1}}$ and 
$\langle\cdot, \cdot\rangle_{\lambda_{2}}$ differ at each point, $w \in M_{\text {red }}$, by a positive multiplicative constant, $\kappa_{i}(w)$, and

$$
\mu_{i, \lambda_{1}}-\mu_{i, \lambda_{2}}=\sqrt{-1} d\left(\partial \log \kappa_{i}(w)\right) ;
$$

hence $\mu_{i, \lambda_{1}}$ and $\mu_{i, \lambda_{2}}$ are canonically cohomologous. Hence, to summarize, the reduced symplectic form, $\omega_{\lambda}$, on $M_{\text {red }}$ satisfies the Duistermaat - Heckman equation:

$$
\omega_{\lambda}=\mu+\sum \lambda_{i} \mu_{i}
$$

where $\mu=\mu_{\lambda}$ is defined by (8.5) and $\mu_{i}=\mu_{i, \lambda}$ is the curvature form of the line bundle $\mathbb{L}_{i}$. These forms are canonically defined 2-forms on $M_{\text {red }}$. Moreover, though they depend on $\lambda$, they are, for different choices of $\lambda$, canonically cohomologous by (8.7) and (8.12).

\section{The Biquard-Gauduchon formula.}

The potential function in formula (8.2):

$$
\rho_{\lambda}=\iota_{\lambda}^{*} \rho-\sum \lambda_{i} \iota_{\lambda}^{*} \log \left|z_{i}\right|^{2}
$$

is not intrinsically defined in terms of $\rho$ since the functions, $z_{i}$, depend upon the choice of the trivialization (8.1). However, there is a simple way to make it intrinsically defined. The function, $\rho_{\lambda}$, lives on $U$, so we can pull it back by the fibration (7.1) to get a function $\pi^{*} \rho_{\lambda}$ on $M_{U}$. We claim that the sum,

$$
\hat{K}_{\lambda}=\pi^{*} \rho_{\lambda}+\sum \lambda_{i} \log \left|z_{i}\right|^{2}
$$

is independent of how we trivialize the bundles, (7.1) over $U$. If one changes this trivialization by replacing $z_{i}$ by $a_{i}(w) z_{i}$ then

$$
\log \left|a_{i}(w) z_{i}\right|^{2}-\pi^{*} \log \iota_{\lambda}^{*}\left|a_{i}(w) z_{i}\right|^{2}=\log \left|z_{i}\right|^{2}-\pi^{*} \log \iota_{\lambda}^{*}\left|z_{i}\right|^{2} ;
$$

so the right-hand side of (9.2) is unchanged. Also note that (8.3) can be written in the more instrinsic form

$$
\pi^{*} \omega_{\lambda}=\sqrt{-1} \partial \bar{\partial} \hat{K}_{\lambda}
$$

In other words, if one has a Kähler potential, $\rho$, on $M$ satisfying $\omega=$ $\sqrt{-1} \partial \bar{\partial} \rho$, one gets an intrinsic Kähler potential, $\hat{K}$, on $M$ satisfying (9.3). Thus, even though there may not be a globally defined Kähler potential for the reduced symplectic form, $\omega_{\lambda}$, there is for its pull-back, $\pi^{*} \omega_{\lambda}$, to $M$. (See [BG], pages 291-293.) 


\section{The Atiyah convexity theorem.}

Our proof of this theorem makes use of some elementary facts about strictly convex functions: Let $V$ be a real vector space and $F: V \rightarrow \mathbb{R}$ a smooth function. We recall that $F$ is strictly convex if the Hessian $d^{2} F_{p}$, is positive definite for all $p \in V$, and is stable if $F(x)$ tends to infinity as $x$ tends to infinity in $V$. Given $\ell \in V^{*}$ let $F_{\ell}(x)=F(x)-\ell(x)$. The stability set of a strictly convex function, $F$, is the set, $S_{F}$, of all $\ell \in V^{*}$ for which $F_{\ell}$ is stable. We will need below the following properties of this set.

Proposition 1. $S_{F}$ is an open convex set.

Proof. Let $\ell=s \ell_{1}+(1-s) \ell_{2}, 0 \leq s \leq 1$. Then if $F_{\ell_{1}}(x)$ and $F_{\ell_{2}}(x)$ tend to $+\infty$ as $x$ tends to infinity so does $F_{\ell}(x)$. To see that $S_{F}$ is open, we may, without loss of generality assume that $\ell=0$ and that $x=0$ is a strict global minimum of $F$. Then we have

$$
F(x)>\min _{\{|x|=1\}}|F(x)|+c \cdot|x|, \text { for }|x|>1,
$$

where $c=\min _{\{|x|=1\}} \frac{\partial F}{\partial r}$, and $c>0$ because $F$ is strictly convex and $F(0)=$ 0 . Then for all $\ell$ such that $|\ell|<c, \ell \in S_{F}$.

Proposition 2. If $F_{1}$ and $F_{2}$ are strictly convex and $F_{1}-F_{2}$ is bounded, $S_{F_{1}}=S_{F_{2}}$.

Proof. $\left(F_{1}\right)_{\ell}(x)$ tends to $+\infty$ as $x$ tends to infinity if and only if $\left(F_{2}\right)_{\ell}(x)$ tends to $+\infty$ as $x$ tends to infinity.

Let $F$ be a function of the form

$$
F(x)=\sum_{i=1}^{N} c_{i} e^{\alpha_{i}(x)}
$$

where $c_{i}>0$ and $\alpha_{i} \in V^{*}$.

Proposition 3. $F$ is stable if and only if zero is contained in the interior of the convex hull of $\left\{\alpha_{i}, i=1, \ldots, N\right\}$. 
Proof. If zero is contained in the exterior or boundary of this set there exists a $\xi \in V$ such that the $\alpha$ 's lie in the half-space

$$
\left\{\mu \in V^{*}, \mu(\xi) \leq 0\right\},
$$

and hence $F(x)$ is bounded along the ray $x=t \xi, t \geq 0$.

Corollary. The stability set of the function $\log F$ is the interior of the convex hull of $\left\{\alpha_{i}, i=1, \ldots, N\right\}$.

Proof. $\log F-\ell=\log F e^{-\ell}$ where

$$
F e^{-\ell}=\sum c_{i} e^{\alpha_{i}-\ell}
$$

and this is stable if and only if $\ell$ is contained in the interior of the convex hull of the $\alpha$ 's.

The Legendre transform of a function, $F$, is the map

$$
L_{F}: V \rightarrow V^{*}, p \rightarrow d F_{p}
$$

Proposition 4. If $F$ is strictly convex, $L_{F}$ maps $V$ bijectively onto $S_{F}$.

Proof. Let $\ell$ be in $S_{F}$. Since $F_{\ell}$ is stable $F_{\ell}(x)$ tends to $+\infty$ as $x$ tends to infinity, hence $F_{\ell}$ has at least one minimum point, $p$. Moreover, since $F$ is strictly convex, every critical point of $F_{\ell}$ is a non-degenerate minimum point. So by the Morse index theorem, $p$ is the unique critical point of $F_{\ell}$; and at $p, d F_{p}=\ell$.

We will now prove that the Atiyah theorem is true in one very simple special case. Let $\tau_{0}$ be a linear action of $T_{\mathbb{C}}$ on $\mathbb{C}^{N+1}$ with weights, $\beta_{i}$, $i=1, \ldots, N+1$ and let $\tau$ be the induced action of $T_{\mathbb{C}}$ on $\mathbb{C} P^{N}$. Let $p$ be the point, $\left[a_{0}, \cdots, a_{n}\right], a_{0}=1$. On the complement of the hyperplane, $z_{0}=0$, the canonical Kähler form, $\omega_{F S}$, is equal to $\sqrt{-1} \partial \bar{\partial} \rho$ where

$$
\rho=\log \left(\frac{\left|z_{0}\right|^{2}+\cdots+\left|z_{n}\right|^{2}}{\left|z_{0}\right|^{2}}\right)
$$

and the restriction of $\rho$ to the $T_{\mathbb{C}}$ orbit through $p$ is the function

$$
\log \left(c_{1} t^{\alpha_{1}}+\cdots+c_{N} t^{\alpha_{N}}+1\right)
$$


where $\alpha_{i}=\beta_{i}-\beta_{0}$ and $c_{i}=\left|a_{i}\right|^{2}$. Making the substitution $t_{i}=e^{s_{i}}, i=$ $1, \ldots, n$ this becomes

$$
F(s)=\log \left(c_{1} e^{\alpha_{1}(s)}+\cdots+c_{N} e^{\alpha_{N}(s)}+1\right) .
$$

Now let $\Phi$ be the moment map associated with the action of $T$ on $\mathbb{C} P^{N}$. By (3.6) the restriction of $\Phi$ to $T_{\mathbb{C}} \cdot p$ is just $L_{F}$; and, by the corollary to proposition 3 , its image is the convex hull of the set consisting of zero and those $\alpha_{i}$ 's for which $c_{i} \neq 0$. In particular this proves

Theorem 10.1. The moment image of $T_{\mathbb{C}} \cdot p$ is a convex polytope.

We will now show that this special case of the convexity theorem implies the convexity theorem in much greater generality: First of all it is clear that if $\tau_{0}$ is a linear action of $T_{\mathbb{C}}$ on $\mathbb{C}^{N+1}$ and $\tau$ is the induced action of $T_{\mathbb{C}}$ on $\mathbb{C} P^{N}$ then, for every non-singular projective variety, $M \subseteq \mathbb{C} P^{N}$, which is invariant under $\tau$, the theorem is true for $T_{\mathbb{C}}$ orbits lying in $M$. Now suppose that $M$ is a compact Kähler manifold whose Kähler form, $\omega$, is an integral form. Then $[\omega]$ is the Chern class of a complex line bundle, $\mathbb{L}$; and, by a theorem of Kodaira, some high power, $\mathbb{L}^{k}$, of $\mathbb{L}$ admits enough global holomorphic sections to imbed $M$ in $\mathbb{C} P^{N}$. Moreover, the action of $T_{\mathbb{C}}$ on $M$ lifts to an action of $T_{\mathbb{C}}$ on $\mathbb{L}$ and hence lifts to a linear action of $T_{\mathbb{C}}$ on the space of holomorphic sections of $\mathbb{L}^{k}$. Thus the Kodaira imbedding intertwines the action of $T_{\mathbb{C}}$ on $M$ with a linear action of $T_{\mathbb{C}}$ on $\mathbb{C} P^{N}$. Let $\gamma$ be this imbedding. The pull-back by $\gamma$ of $\omega_{F S}$ is not the Kähler form on $M$; however by Kodaira's theorem $k[\omega]=\gamma^{*}\left[\omega_{F S}\right]$. (For simplicity, we will assume $k=1$.) Hence there exists a $T$-invariant function, $H: M \rightarrow \mathbb{R}$ satisfying

$$
\omega=\gamma^{*} \omega_{F S}+\sqrt{-1} \partial \bar{\partial} H .
$$

Now let $q$ be a point in $M$ and let $p=\gamma(q)$. Then by (10.3) the restriction of $\omega$ to the orbit, $T_{\mathbb{C}} \cdot q$ is equal to $\sqrt{-1} \partial \bar{\partial} F_{1}$ where

$$
F_{1}\left(s_{1}, \ldots, s_{n}\right)=H\left(s_{1}, \ldots, s_{n}\right)+F\left(s_{1}, \ldots, s_{n}\right)
$$

$F$ being the function (10.2). However, $H$ is bounded on $T_{\mathbb{C}} \cdot q$ so by proposition $2 L_{F}$ and $L_{F_{1}}$ have the same image. Thus we can conclude:

Theorem 10.2. The moment image of $T_{\mathbb{C}} \cdot q$ is identical with the moment image of $T_{\mathbb{C}} \cdot p$ (and hence, in particular, by theorem 10.1, is a convex polytope). 


\section{The symplectic quotient of a Kähler-Einstein manifold.}

Suppose that the Kähler form on $M$ is Kähler-Einstein, i.e., that the Ricci form and the Kähler form satisfy $\mu=\kappa \omega, \kappa \in \mathbb{R}$. We will show that the reduced Kähler form, $\omega_{\lambda}$, and reduced Ricci form, $\mu_{\lambda}$, satisfy (1.1). It is enough to prove this identity on open pseudoconvex sets, $U$, of $M_{\text {red }}$ over which $M_{\text {stable }}$ is the trivial bundle: $\left(\mathbb{C}^{*}\right)^{n} \times U$. Let $\rho$ be a potential function on $U$ of the form (2.3) such that $\omega=\sqrt{-1} \partial \bar{\partial} \rho$ and such that the moment map (3.1) is identical with the given moment map. Then if $h$ is the Ricci potential associated with $\rho, \kappa \rho-h$ is pluri-harmonic so by theorem 2.2

$$
\kappa \rho=h+\sum a_{i} \log \left|z_{i}\right|^{2}+\operatorname{Re} f
$$

$f \in \mathcal{O}(U)$. Thus with $c_{i}=\left(a_{i}-1\right)$ the expression

$$
\kappa\left(\iota_{\lambda}^{*}\left(\rho-\sum \lambda_{i} \log \left|z_{i}\right|^{2}\right)+\sum \lambda_{i} \log \iota_{\lambda}^{*}\left|z_{i}\right|^{2}\right)
$$

is equal to the expression

$$
\iota_{\lambda}^{*}\left(h+\sum \log \left|z_{i}\right|^{2}\right)+2 \log V_{\text {eff }}+\operatorname{Re} f
$$

plus the expression

$$
\sum c_{i} \log \iota_{\lambda}^{*}\left|z_{i}\right|^{2}-2 \log V_{\text {eff }}
$$

Applying $\partial \bar{\partial}$ to these three expressions and using (6.12) one gets (1.1).

We will next show that the $c_{i}$ 's are given by the divergence equations (1.2). Differentiating the identity (11.1) with respect to the vector field, $Z_{i}=z_{i} \partial / \partial z_{i}$, we get

$$
\kappa Z_{i} \rho=Z_{i} h+a_{i}
$$

But the symplectic volume form, $\omega^{d}$, is, up to a constant multiple, equal to

$$
e^{h} \prod d z_{i} \wedge d \bar{z}_{i} \prod d w_{\alpha} \wedge d \bar{w}_{\alpha}
$$

Hence

$$
\operatorname{div} Z_{i}=L_{Z_{i}} \omega^{d} / \omega^{d}=Z_{i} h+1
$$

and by (3.1)

$$
\phi_{i}=Z_{i} \rho,
$$

so (11.5) reduces to

$$
\kappa \phi_{i}=\operatorname{div} Z_{i}+c_{i} .
$$


Note in particular that if the manifold, " $M$ ", in this section is as in section 7

the open dense subset, $M_{\text {stable }}$, of a compact manifold, $M$, we can integrate (1.2) over $M$, and using the fact that the integral

$$
\int\left(\operatorname{div} Z_{i}\right) \omega^{d}=\int d \iota\left(Z_{i}\right) \omega^{d}
$$

is zero, get

$$
\kappa \int \phi_{i} \omega^{d}=c_{i} \operatorname{vol}(M)
$$

Thus if we normalize the moment map by requiring, as in section 7 , that the integral on the left be zero, we end up with $c_{i}=0$, and the equation (1.1) reduces to the simpler form

$$
\mu_{\lambda}-2 \sqrt{-1} \partial \bar{\partial} \log V_{\text {eff }}=\kappa\left(\omega_{\lambda}+\sum \lambda_{i} \mu_{i}\right)
$$

If we restrict to the case of level 0 , i.e., $\lambda=0$, we reproduce an earlier result of Futaki's [Fu], Theorem 7.3.2, in the case of a Kähler-Einstein manifold $M$. Futaki treats the case of $M$ a Fano manifold, which would be treated here in the same fashion, mutatis mutandis.

\section{Kähler metrics on toric varieties.}

Let $\omega$ be the standard Kähler form on $\mathbb{C}^{n}$ :

$$
\omega=\sqrt{-1} \partial \bar{\partial} \rho
$$

where $\rho=\sum\left|z_{i}\right|^{2}$. The linear action of $T=\left(S^{1}\right)^{n}$ on $\mathbb{C}^{n}$ is Hamiltonian with respect to this form, and by (3.1), the moment map associated with the potential, $\rho$, is

$$
\Phi(z)=\left(\left|z_{1}\right|^{2}, \ldots,\left|z_{n}\right|^{2}\right) .
$$

Now let $G$ be a subtorous of $T$ and $\mathfrak{g}$ its Lie algebra. From the inclusion of $G$ in $T$, one gets an inclusion of $\mathfrak{g}$ into $\mathbb{R}^{n}$ and a transpose map

$$
L: \mathbb{R}^{n} \rightarrow \mathfrak{g}^{*}
$$

and the moment map associated with the action of $G$ on $\mathbb{C}^{n}$ is just the composition of (12.1) and (12.2). It is easy to see that the images of the standard basis vectors, $e_{i}$, of $\mathbb{R}^{n}$ are the weights, $\alpha_{i}$, of the representation of $G$ on $\mathbb{C}^{n}$, so by (12.1) and (12.2) this $G$-moment map can be written

$$
\psi(z)=\sum\left|z_{i}\right|^{2} \alpha_{i}
$$


This is a proper mapping if and only if the $\alpha_{i}$ 's are polarized, i.e., if and only if there exists a $\xi \in \mathfrak{g}$ such that $\alpha_{i}(\xi)>0$ for all $i$. We will assume henceforth that this condition holds.

Now let $\lambda$ be a regular value of $\psi$ and assume that $G$ acts freely on the level set, $\psi^{-1}(\lambda)$. Then the reduced space, $M_{\lambda}=\psi^{-1}(\lambda)$ is a Kähler manifold with Kähler form, $\omega_{\lambda}$. Moreover, since the action of $T$ on $\mathbb{C}^{n}$ commutes with the action of $G$, one gets an induced Hamiltonian action of $T$ on $M_{\lambda}$ with moment map, $\Phi_{\lambda}: M_{\lambda} \rightarrow \mathbb{R}^{n}$. Moreover, if $p$ is the projection of $\psi^{-1}(\lambda)$ onto $M_{\lambda}$

$$
\Phi_{\lambda} \circ p=\Phi \circ \iota_{\lambda}
$$

and this equation completely determines $\Phi_{\lambda}$.

The moment polytope of the Hamiltonian $T$-manifold, $M_{\lambda}$, is by definition the image of $M_{\lambda}$ with respect to this moment map. By (12.4) this is equal to the image with respect to $\Phi$ of the set, $\psi^{-1}(\lambda)$; and by (12.1) and (12.2) this is equal to the set

$$
\Delta_{\lambda}=\left\{\left(t_{1}, \ldots, t_{n}\right) \in \mathbb{R}_{+}^{n}, \sum t_{i} \alpha_{i}=\lambda\right\} .
$$

In other words it is the intersection

$$
\Delta_{\lambda}=\mathbb{R}_{+}^{n} \cap L^{-1}(\lambda)
$$

of the positive orthant in $\mathbb{R}^{n}$ with the affine subspace, $L^{-1}(\lambda)$. It is easy to see that $\lambda$ is a regular value of $\psi$ if and only if $L^{-1}(\lambda)$ intersects the faces of $\mathbb{R}_{+}^{n}$ transversally. Thus the codimension $\kappa$ faces of $\Delta_{\lambda}$ are the intersections of $L^{-1}(\lambda)$ with the codimension $\kappa$ faces of $\mathbb{R}_{+}^{n}$. In particular the facets (codimension one faces) are the intersections of $L^{-1}(\lambda)$ with the facets, $t_{i}=$ 0 , of $\mathbb{R}_{+}^{n}$; so if $j: \Delta_{\lambda} \rightarrow \mathbb{R}^{n}$ is the inclusion map, the function

$$
j^{*} t_{i}=: \ell_{i}
$$

is just the distance function on $\Delta_{\lambda}$ to the $i^{\text {th }}$ facet. Now let $U$ be the open dense subset

$$
\left(\mathbb{C}^{*}\right)^{n} \cap \psi^{-1}(\lambda) / T
$$

of $M_{\lambda}$ and fix a base point, $c=\left(c_{1}, \ldots, c_{n}\right)$, in $\Delta_{\lambda}$.

Theorem 12.1. The restriction of $\omega_{\lambda}$ to $U$ is equal to $\sqrt{-1} \partial \bar{\partial} \rho_{\lambda}$ where $\rho_{\lambda}$ is the pull-back by $\Phi_{\lambda}$ of the function

$$
\sum \ell_{i}-c_{i} \log \ell_{i}
$$


Proof. On $\left(\mathbb{C}^{*}\right)^{n}$ the potential function

$$
\rho_{1}=\sum\left|z_{i}\right|^{2}-c_{i} \log \left|z_{i}\right|^{2}
$$

satisfies $\sqrt{-1} \partial \bar{\partial} \rho_{1}=\omega$; and by (3.3), the $T$-moment map associated with this potential is $\Phi-c$. Thus the $G$-moment map associated with this potential is $L$ composed with $\Phi-c$, or $\psi-\lambda$. In particular, the zero level set of this moment map is the $\lambda$-level set of $\psi$ and so, by (4.6), one gets the induced Kähler form on $M_{\lambda}$ by applying $\sqrt{-1} \partial \bar{\partial}$ to the funciton

$$
\iota_{\lambda}^{*}\left(\sum\left|z_{i}\right|^{2}-c_{i} \log \left|z_{i}\right|^{2}\right)
$$

and by (12.4) and (12.6) this is just the function

$$
\Phi_{\lambda}^{*}\left(\sum \ell_{i}-c_{i} \log \ell_{i}\right)
$$

As we mentioned in the introduction, the proof above of theorem 12.1 is a slightly simplified version of the proof described in [CDG]. For applications of this theorem, see $[\mathrm{Ab}]$ and $[\mathrm{CDG}]$.

\section{References.}

[AM] R. Abraham and J. Marsden, Foundations of Mechanics, BenjaminCummings, Reading, MA (1978).

[Ab] Miguel Abreu, "Kähler geometry of toric varieties and extremal metrics", Int. J. Math. 9 (1998), 641-651.

[At] M. Atiyah, "Convexity and commuting Hamiltonians", Bull. Lond. Math. Soc. 14 (1981), 1-15.

[Be] A.L.Besse, Einstein Manifolds, Ergeb. Math. Grenzgb. volume 10, Springer Verlag, Berlin, Heidelberg, New York (1987).

[BG] O. Biquard and P. Gauduchon, "Hyper-Kähler metrics on cotangent bundles of Hermitian symmetric spaces", Lecture notes in pure and applied mathematics 184 Dekker, Amsterdam (1997).

[BD] D. Burns and P. DeBartolomeis, "Stability of vector bundles and extremal metrics", Invent. Math. 92 (1988), 403-407. 
[CDG] D. Calderbank, L. David and P. Gauduchon, "About the Guillemin formula for the Kähler potential of a toric manifold", preprint.

[DH] J.J. Duistermaat and G.L. Heckman, "On the variation in cohomology of the symplectic form of the reduced phase spce", Invent. Math. 69 (1982), 259-269.

[Fu] A. Futaki, Kähler-Einstein Metrics and Integral Invariants, Lecture Notes in Mathematics 1314, Springer Verlag, Berlin, Heidelberg, New York (1988).

[Gu] V. Guillemin, "Kähler structures on toric varieties", J. Diff. Geom. 40 (1984), 285-309.

[HH1] P. Heinzner and A. Huckleberry, "Kählerian potentials and convexity properties of the moment map", Invent. Math. 126 (1996), 65-84.

[HH2] ___-_, ___, "Analytic Hilbert quotients", Several Complex Variables, Math. Sci. Res. Inst. Publ. 37, Cambridge Univ. Press (1999), 309349 .

[HH3] ____, ___, "Kählerian structures on symplectic reductions", in Complex Analysis and Algebraic Geometry (A Volume in Memory of Michael Schneider), ed. T. Peternell and F-O. Schreyer, de Gruyter Verlag (2000), 226-253.

[HHL] ___, ___, F. Loose, "Kählerian extensions of the symplectic reduction", J. Reine und Angew. Math. 455 (1994), 123-140.

[HL] __., F. Loose, "Reduction of complex Hamiltonian G-spaces", Geom. and Func. Anal. 4 (1994), 288-297.

[L] C. LeBrun, "Explicit self-dual metrics on $\mathbb{C P}^{2} \# \cdots \mathbb{C P}^{2}$ ", J. Diff. Geom. 34(1991), 223-253.

[MFK] D. Mumford, J. Fogarty and F. Kirwan, Geometric Invariant Theory, Springer, Berlin, Heidelberg, New York (1991).

[PP] H. Pedersen and Y.-S. Poon, "Hamiltonian constructions of KählerEinstein metrics and Kḧler metrics of constant scalar curvature", Comm. Math. Phys. 136 (1991), 309-326.

[S] R. Sjamaar, "Holomorphic slices, symplectic reduction and reduction and multiplicities of representations", Ann. Math. 134 (1995), 87129. 
D. Burns

M.I.T., Cambridge, MA and Univ. of Michigan, Ann Arbor, MI dburns@umich.edu

V. Guillemin

M.I.T., CAmbridge, MA

vwg@math.mit.edu 
